\title{
Report
}

\section{Epidemiological Profile of Thrombophilia in Marrakech (Morocco): About 200 Cases}

\section{Ilham Karrati, Hanane Mouhib, Hicham Yahyaoui, Radia Amaddah, Mustapha Aitameur, Mohamed Chakour}

Haematology Laboratory of Avicenne Military Hospital, University Hospital Mohamed VI, Marrakech, Morocco

\author{
Email address: \\ ilhamkarrati@gmail.com (I. Karrati),mouhib.hanane1@gmail.com (H. Mouhib),hichamyahyaouik@gmail.com (H. Yahyaoui), \\ radiat.sa@gmail.com (R. Amaddah), aitameur.mustapha@yahoo.com (M. Aitameur),mchakour2005@yahoo.fr (M. Chakour) \\ *Corresponding author
}

\section{To cite this article:}

IlhamKarrati, HananeMouhib, HichamYahyaoui, RadiaAmaddah, MustaphaAitameur, MohamedChakour. Epidemiological Profile of Thrombophilia in Marrakech (Morocco): About 200 Cases. American Journal of Laboratory Medicine. Vol. 4, No. 5, 2019 , pp. $79-86$. doi: 10.11648/j.ajlm.20190405.11

Received: July 31, 2019; Accepted: August 30, 2019; Published: September 19, 2019

\begin{abstract}
Thrombophilia is a situation characterized by an increased tendency to thrombosis. The main objective of this work is to report on the experience of the Haematology Laboratory of the Avicenne Military Hospital of Marrakech, through a retrospective descriptive study carried out over a period of 9 years, on 200 requests for a thrombophilia check-up; and secondly, to discuss its indications and interest in the etiological diagnosis of unexplained thrombosis in the light of the latest recommendations. The thrombogenic risk factor most commonly found in our study was PS deficiency, in contrast to the predominance of Factor V mutation in Western countries, hence the importance of emphasizing that in clinical practice, the first-line thrombophilia assessment should always combine, in accordance with the latest recommendations: inhibitor deficiencies, F V and F II mutations, antiphospholipid antibodies and possibly F VIII determination.
\end{abstract}

Keywords: Thrombophilia Assessment, Protein C, Protein S, Antithrombin, Factor V mutation, Factor II Mutation

\section{Introduction}

Thrombophilia is a state of hypercoagulability that predisposes to thrombotic events. We distinguish between constitutional and acquired thrombophilia [1-2].

The constitutional thrombophilia assessment traditionally looks for deficiencies in coagulation inhibitors including antithrombin, protein $\mathrm{C}$ and protein $\mathrm{S}$, as well as the Leiden mutation of factor $\mathrm{V}$ and the G20210A mutation of prothrombin [3].

Obviously, the search for these constitutional anomalies must not hide the need to search for acquired pathologies such as the presence of cancer or antiphospholipid syndrome, and secondly, other less frequent etiologies whose relevance is often debated such as factor VIII elevation, dysfibrinogenesis, fibrinolytic system anomalies and hyperhomocysteinemia [4-5].

The main objective of this work was to determine the epidemiological profile of thrombophilia in the region of Marrakech, and secondly to discuss the indications for the thrombophilia balance and its interest in the etiological diagnosis of unexplained thrombosis in the light of the latest recommendations.

\section{Patients and Methods}

Our work is a retrospective study, descriptive over a period of 9 years, from 2010 to 2018, on 200 requests for thrombophilia screening, received at the Laboratory of Hematology at the Avicenne Military Hospital in Marrakech.

Included in our study were all patients who had a biological check-up for thrombophilia following a venous thrombotic episode, unexplained arterial thrombosis, repeated fetal loss and risk situations (pregnancy with a history of venous thrombosis).

The informations were collected from the medical files and 
processed using a farm return.

Data entry and analysis was performed using EXCEL software and a descriptive method using simple variables such as percentages and averages.

Samples were taken in accordance with the conditions of the pre-analytical phase. The various parameters were measured using calibrated and controlled automatons; thus the measurement of protein $\mathrm{C}(\mathrm{PC})$, protein $\mathrm{S}$ (PS), antithrombin (AT) and factor VIII activity was done by chronometric method. The search for the mutation of factor $\mathrm{V}(\mathrm{FV})$ and factor II (FII) was carried out by PCR technique. The detection of anti-phospholipid antibodies (APL) was performed by measuring diluted Russel Viper Venom Time and calculating the Rosner Index.

\section{Results}

\subsection{Age and Gender}

Over the study period, we collected 200 cases. The average age of the patients was 39 years with extremes ranging from 3 to 60 years and a predominance of the age group under 45 years. 95 patients were male (47.5\%) compared to 105 female $(52.5 \%)$ with an $\mathrm{M} / \mathrm{F}$ sex ratio of 0.9 . The majority of requests for thrombophilia screening came from hospitalizations in different departments ( $84 \%$ of cases) (Figure 1).

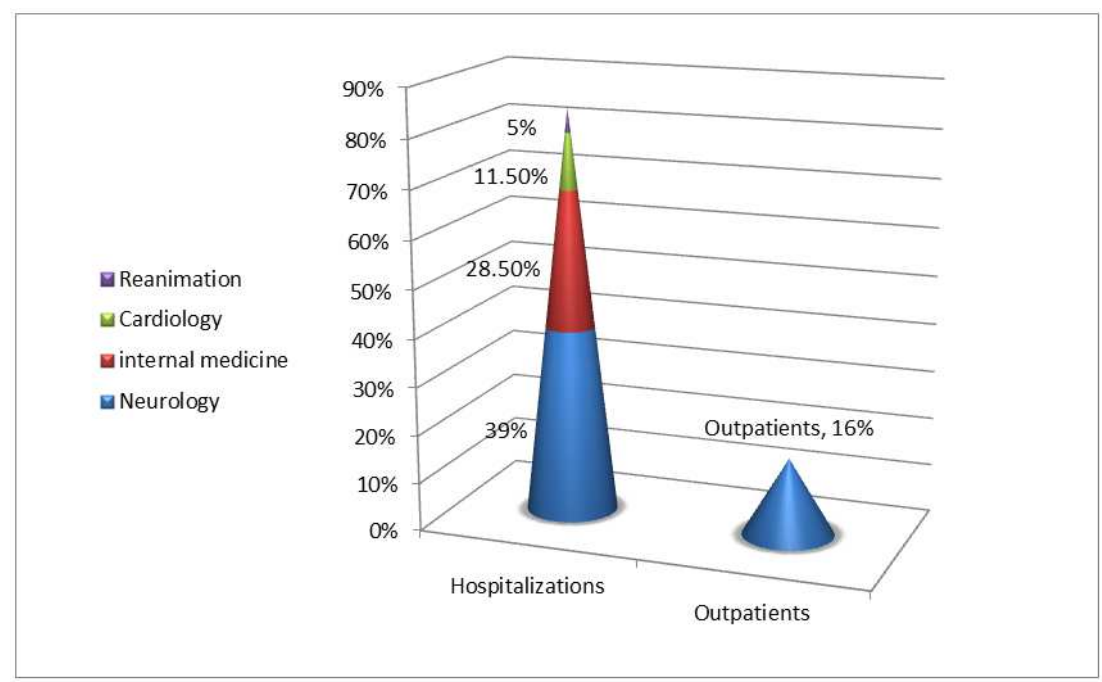

Figure 1. Distribution of balance sheets according to requesting services.

\subsection{The Circumstances of the Diagnosis}

The main diagnostic circumstances that motivated the request for a check-up were deep veinous thrombosis, which represented $45.5 \%$, followed by ischemic stroke in $29.5 \%$ of cases, followed by pulmonary embolism, cerebral thrombophlebitis and repeated miscarriages, which represented $10 \%, 9 \%$ and $6 \%$ of requests respectively (Figure 2 ).

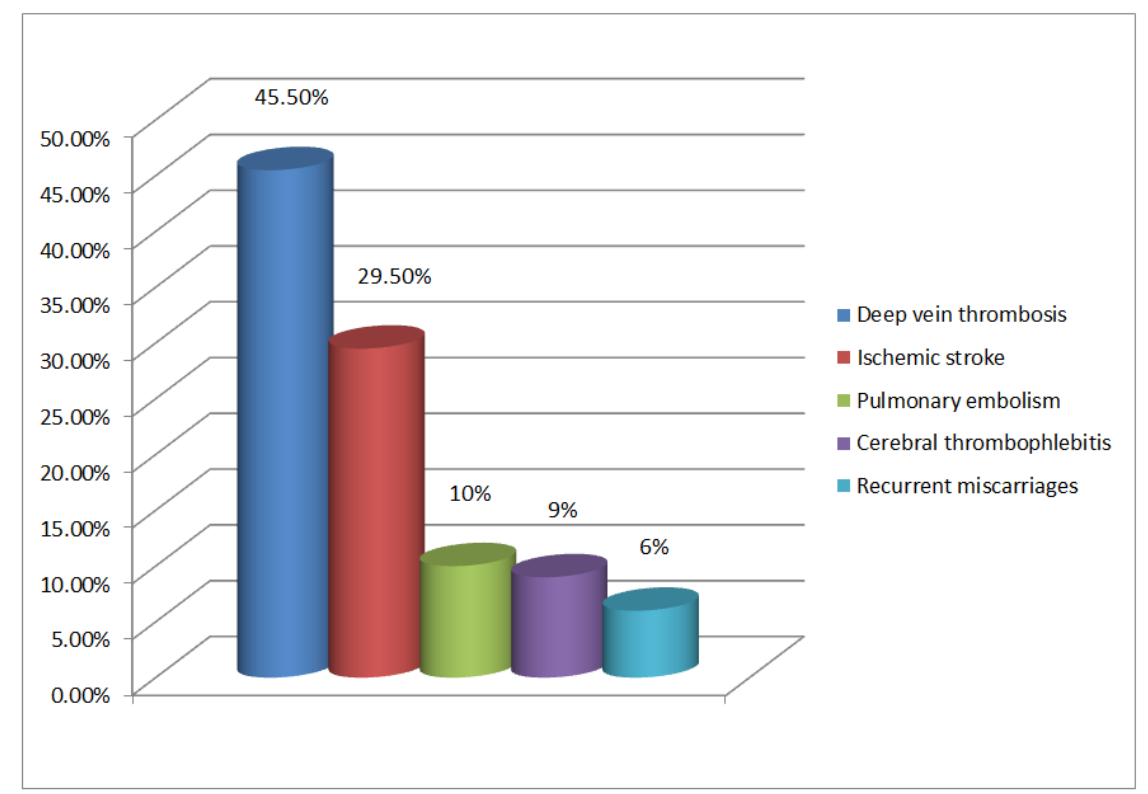

Figure 2. Distribution of patients by diagnosis. 


\subsection{Distribution of the Requested Reports}

Various tests were prescribed, the balance combining the search for the activity of protein $\mathrm{C}$, protein $\mathrm{S}$ and antithrombin was the most requested (64.5\%). Figure 3 shows the distribution of the different balance sheets requested.

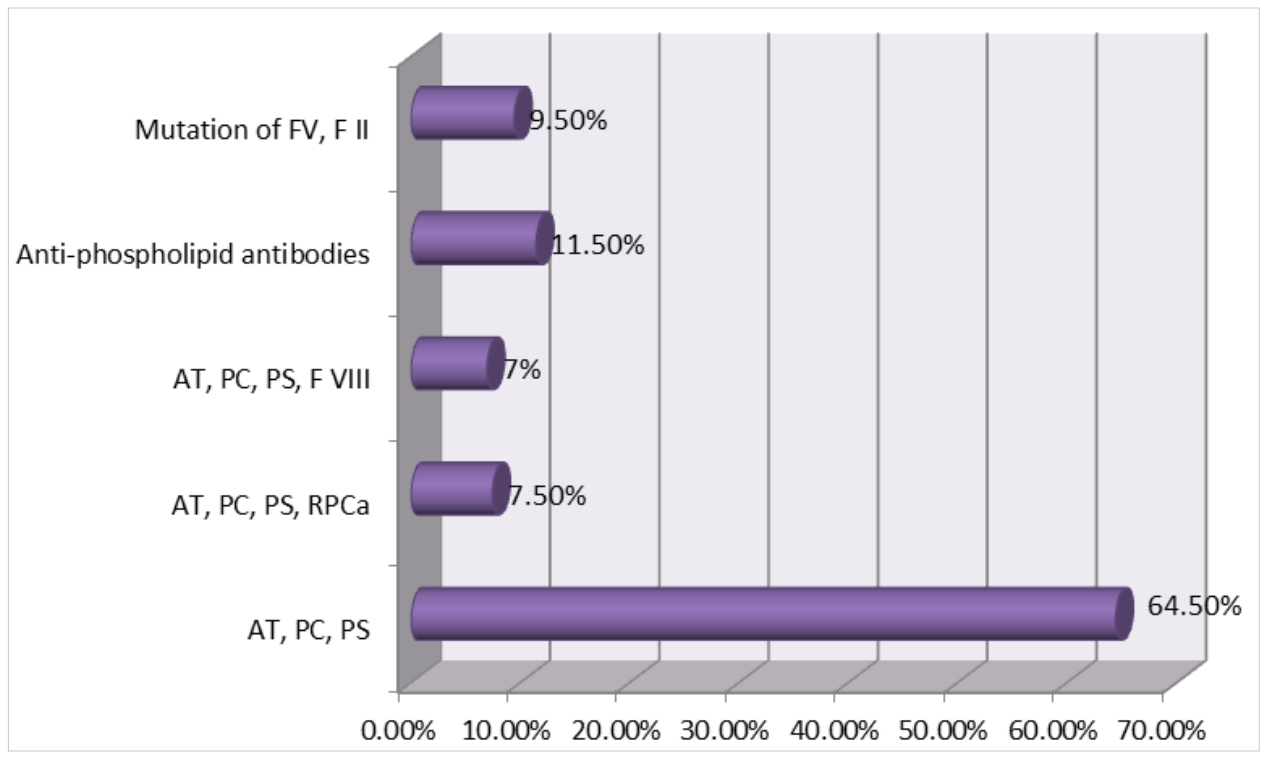

Figure 3. Distribution of the completed balance sheets and their percentages.

\subsection{Distribution of Biological Anomalies}

Among the 200 tests carried out, 91 tests or $45.5 \%$ were positive, divided into several anomalies: the isolated deficit in PS ranked first with 28 cases $(30.7 \%)$, followed by the presence of anti phospholipid antibodies with 16 cases (17.6\%), the isolated deficit in PC with 12 cases (13.2\%), combined protein $\mathrm{C}$ and $\mathrm{S}$ deficiency with 11 patients (12.1\%), F VIII elevation with 7 patients, Antithrombin deficiency with 5 patients, factor $\mathrm{V}$ mutation with 5 patients also, combined AT, PC and PS deficiency with 4 patients, while factor II mutation represented only $3 \%$ of all abnormalities (Figure 4).

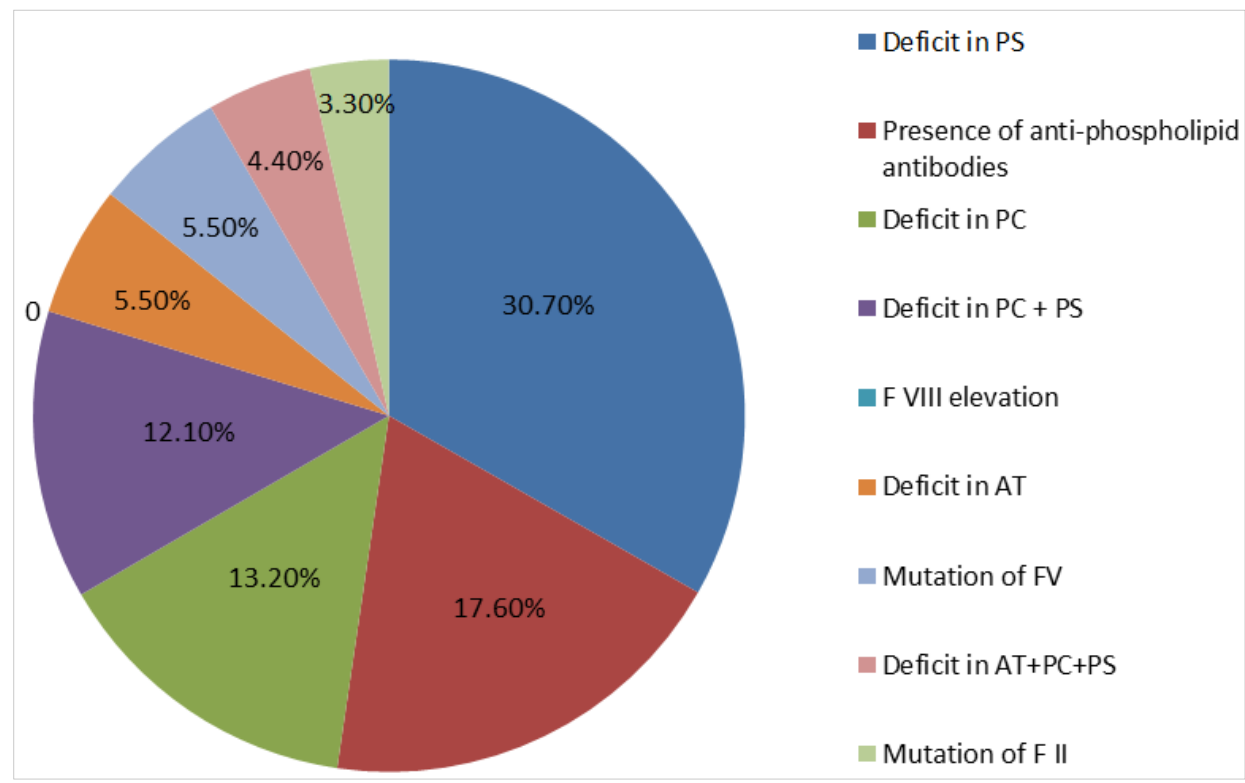

Figure 4. Distribution of biological anomalies found in our series.

\subsection{Distribution of Anomalies According to Diagnosis}

Out of 129 reports sent following venous thrombosis, 59 were positive or $45.7 \%$. The most frequent abnormalities were combined PC and PS deficiency and the presence of
APLantibodies in 11 patients (18.7\%), followed by PS deficiency (13.5\%), PC deficiency (13.5\%) and FVIII elevation (12\%); AT deficiency and FII mutation were the least frequent and were only recorded in 3 patients (Figure 5). 


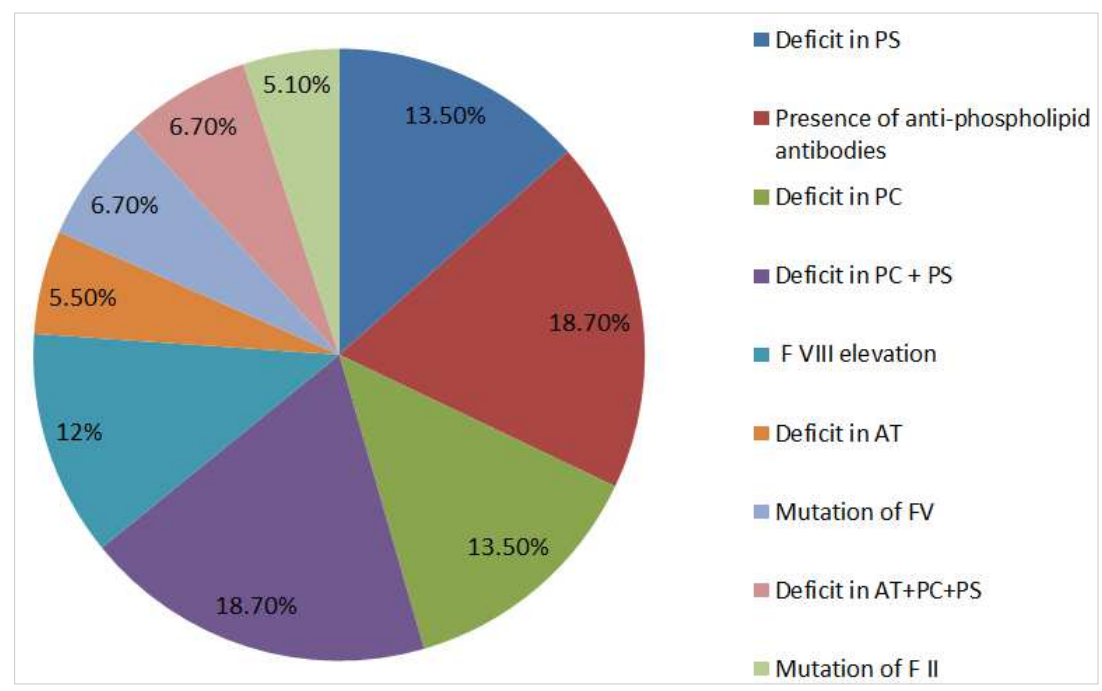

Figure 5. Distribution of anomalies for venous thrombosis.

In addition, among the 59 requests received following ischemic stroke, 24 were positive or $40.6 \%$; divided into 20 cases of isolated PS deficiency (83.3\%) and 4 cases of isolated PC deficiency (16.6\%) (Figure 6).

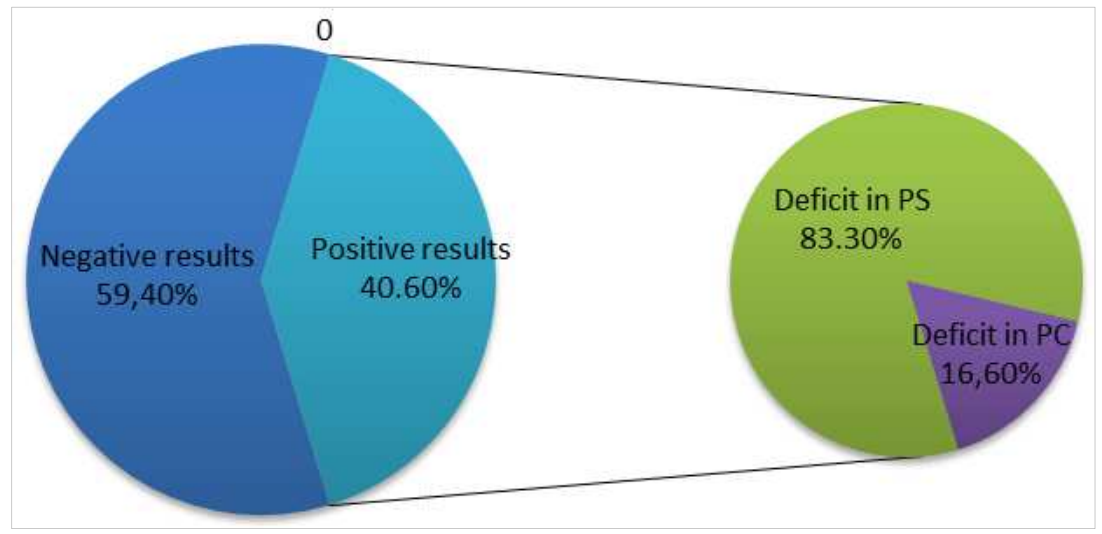

Figure 6. Distribution of anomalies for ischemic strokes.

As for the thrombophilia test motivated by repeated miscarriages in 12 patients, it was positive in 8 cases, 5 of which had the presence of APL antibodies, two had an AT deficiency and one had a F V Leiden mutation (Figure 7).

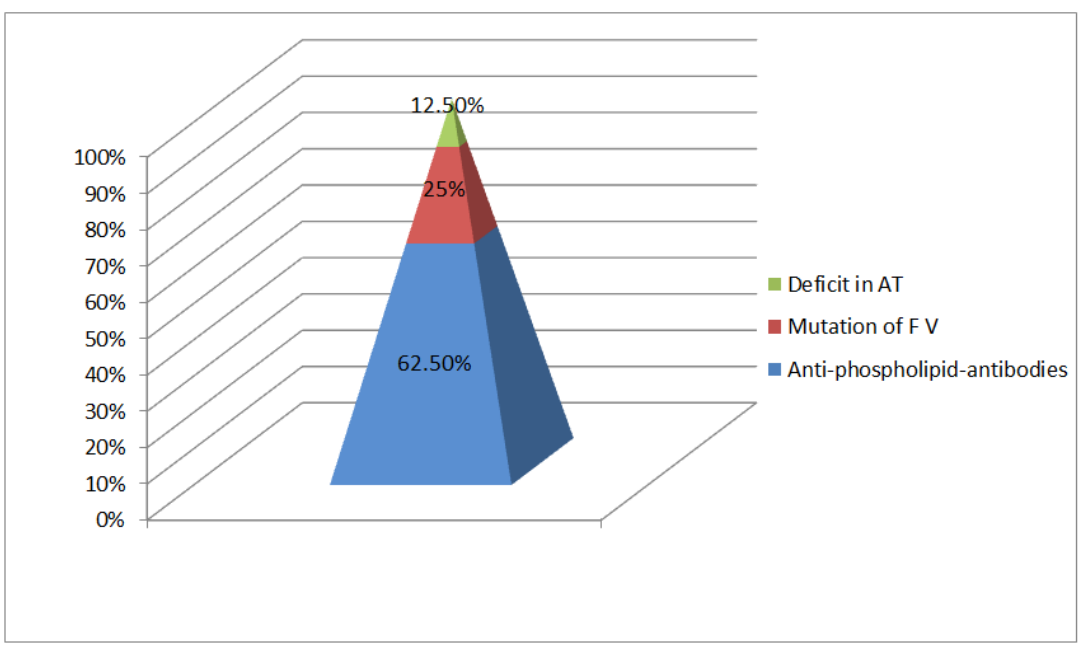

Figure 7. Distribution of anomalies for repeated miscarriages. 


\section{Discussion}

\subsection{The Age}

According to the literature; the notion of thrombophilia is applied to thromboses occurring before the age of 45 . In our study, the age of the patients was around 39 years and $70 \%$ were under 45 years of age, which is consistent with the literature [1-2].

\subsection{The Gender}

Gender is not involved in the determinism of thrombophilia, studies mainly assess the effect of gender on the risk of recurrence [3]. In our study, there is a slight female predominance with a sex ratio of 0.9 .

\subsection{The Time of the Thrombophilia Check-Up}

The demand for thrombophilia screening during patient hospitalization was $84 \%$ compared to only $16 \%$ from outpatient clinics. Knowing that tests should not be performed at the acute phase of thrombosis due to the patient's inflammatory status and the initiation of anticoagulant therapy, false positive results may lead to the diagnosis of a deficiency that the patient may not have, and normal results may provide false confidence; this results in repeated tests and unnecessary cost increases. Although the polymerase chain reaction (PCR) test for factor V Leiden mutation and G20210A prothrombin gene mutation is reliable in any clinical setting, it is not necessary to request thrombophilia screening tests in the emergency department or during hospitalization for an acute thrombotic event, as the initial management will not change following such tests [4].

\subsection{Indications of the Thrombophilia Test}

Currently, learned societies are in constant debate about the relevance of performing a thrombophilia assessment in venous thromboembolic disease (VTED); with recommendations in recent years ranging from no assessment or a very limited assessment by the British Committee for Standards in Hematology to much more permissive recommendations by the
International Consensus Statement [4-5].

In France, the Study Group on Hemostasis and Thrombosis and the French Society of Vascular Diseases has issued recommendations proposing to carry out a thrombophilia assessment in the event of a first episode of VTED (proximal deep vein thrombosis and/or pulmonary embolism) idiopathic before the age of 60. The European Society Of Cardiology considers an event to be idiopathic in the absence of provoking factors such as surgery, trauma, immobilization, pregnancy, taking oral contraception or hormone replacement within 6 weeks to 3 months before diagnosis [6-7].

Currently, all the data tend to limit the thrombophilia assessment to very specific situations. Recommendations from the British Society of Hematology have been published, integrating epidemiological elements and their therapeutic implications, and defining three groups of patients for whom thrombophilia research is discussed [8] (Table 1). Indeed, the interest of the thrombophilia assessment is clearly relevant in the case of pulmonary embolism or proximal deep vein thrombosis (DVT), whose potential severity and risk of recurrence are known; but it is more difficult to decide when it comes to less serious events, such as distal vein thrombosis or superficial venous thrombosis. The probability of finding thrombophilia appears to be lower with leg DVT, according to the results of two retrospective studies by Caprini et al; $42 \%$ of patients with distal vein thrombosis had thrombophilia compared to $61 \%$ of all patients with DVT [9]. In the study by Martinelli et al; the proportion of distal vein thrombosis was $6 \%$ in patients with a Leiden factor $\mathrm{V}$ mutation, $7 \%$ in patients with a prothrombin gene mutation and $16 \%$ in patients without thrombophilia [10]. In this context, it is not clear what a search for constitutional thrombophilia could lead to a decision on the duration of treatment whose necessity is not clearly demonstrated. On the other hand, the search for antiphospholipid antibodies could be useful, even in cases of distal vein thrombosis, as their detection would lead to the long-term continuation of anticoagulants [11].

Table 1. Indications of the thrombophilia assessment [8].

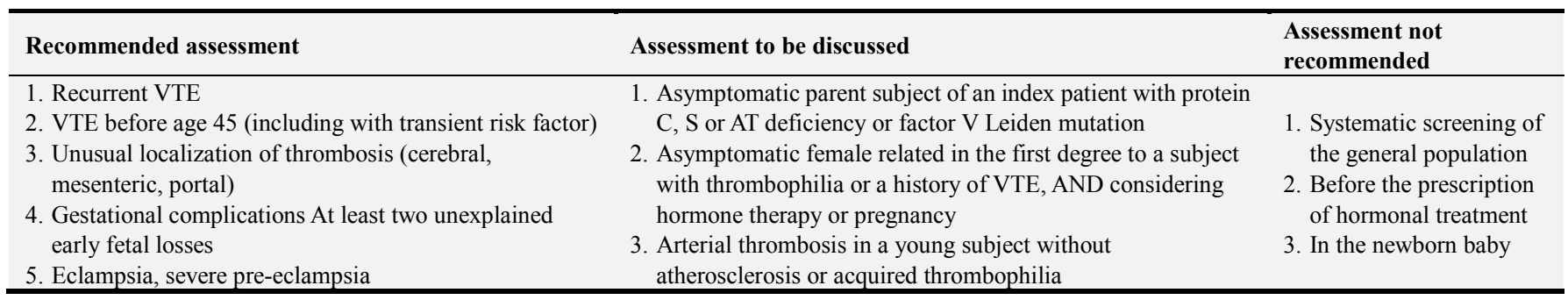

In our study; the majority of thrombophilia check-up prescriptions (45.5\%) were following DVT (91 patients), which is consistent with the literature. $29.5 \%$ were requested following arterial thrombosis, $10 \%$ following pulmonary embolism, $9 \%$ following cerebral thrombophlebitis and only
$6 \%$ or 12 cases of repeated miscarriages, whereas current data in the literature suggest that women with a history of obstetric complications without obvious cause should have a hemostasis test even in the absence of a personal and/or family thrombotic history. In a study of 494 patients at the 
University Hospital of Reindeer, $45.5 \%$ of the assessments were referred for venous thrombosis, $13.2 \%$ for arterial thrombosis and $11.7 \%$ for gynaeco-obstetrical complications [12].

\subsection{The Abnormalities to Be Investigated During Thrombophilia}

The search for thrombophilia, when recommended, should focus on all the anomalies responsible, as there is no clinical characteristic to guide us towards a particular anomaly. This assessment should be carried out ideally before starting anticoagulant treatment or at a distance (one month) from stopping it. Indeed, antivitamins $\mathrm{K}$ disrupt the dosages of proteins $\mathrm{C}$ and $\mathrm{S}$, and heparin reduces the antithrombin. Abnormalities found during hormonal treatment or gestation should be monitored remotely. The first step consists of performing a blood count for an acquired abnormality, an inflammatory test, the search for a lupus anticoagulant and anticardiolipin antibodies. The search for a constitutional anomaly according to a recent consensus will only include the search for a deficiency in coagulation inhibitors by phenotypic determination of AT, PC and PS, and by genotypic testing for mutations in factor V Leiden and factor II; however, this test for a constitutional anomaly as described, is positive in only $50 \%$ of cases. Other biological abnormalities have been investigated and identified; among them factor VIII elevation and hyperhomocysteinemia, their systematic investigation was not recommended at first intention due to their low prevalence [13] (Table 2).

Table 2. Recommended thrombophilia check-ups [13].

\begin{tabular}{|c|c|c|}
\hline First-line thrombophilia research & Search for other acquired thrombophilias & Second-line assessment \\
\hline 1. Search for TA deficiency (activity); & yeloproliferative syndrome, & 1. Dysfibrinogenesis (mutation \\
\hline 2. Search for protein C deficiency (activity); & drome, inflammatory bowel & search); \\
\hline 3. Search for protein $\mathrm{S}$ deficiency (activity and free protein $\mathrm{S}$ ); & diseases, connectivity; & 2. Search for the C677T MTHFR \\
\hline 5. Search for the G20210A mutation of factor II; & proteinuria research, NFS, immunological & 3. Determination of the activity of \\
\hline 6. Search for the mutation factor V Leiden); & assessment and imaging, JAK mutation & IX and XI \\
\hline 7. Search for antiphospholipid antibodies. & research according to clinical orientation. & 4. Homocysteinemia determination. \\
\hline
\end{tabular}

In our study, various tests were requested. The most frequently prescribed assessment included only the search for the activity of CP, PS and AT (64.5\%). Antiphospholipid antibodies were requested in $11.5 \%$ of cases, the search for the mutation of F V and F II was $9.5 \%$ of cases, the balance combining the determination of the three coagulation inhibitors with the determination of factor VIII and the search for the mutation of $\mathrm{F} \mathrm{V}$ represented only $7 \%$ and $7.5 \%$ respectively of all requests.

In our series, $45.5 \%$ of patients were diagnosed with a biological abnormality; this is consistent with the literature, where it is reported that 30 to $50 \%$ of deficits are present in patients with at least one thrombotic episode [14-15].

\subsubsection{Antithrombin Deficiency}

Constitutional deficiencies in AT are due to genetic abnormalities defining two types of deficits: quantitative type I deficiency and qualitative type II deficiency, during which the protein is normally secreted but with functional abnormalities, with low levels by chromogenic method and normal by immunological method. In addition; AT deficiency may be due to acquired disorders such as: hepatocellular insufficiency disseminated intravascular coagulation (DIC), nephrotic syndrome, in which case the deficiency is reversible upon treatment of the underlying etiology [16]. Among the subjects who present a thrombophilia table, an AT deficiency is found in 0.5 to $4.9 \%$ of cases, which was the case in our study; Out of 200 cases, 9 patients had an AT deficiency, or $4.5 \%$ of cases.

\subsubsection{The Deficit in PC and/or PS}

Deficiencies in PC and/or PS may be of genetic origin, quantitative (type I 90\%) with normal activity and low concentration, or qualitative (type II 10\%) with normal concentration and low activity, or acquired as a result of hepatocellular failure, DIC, AVK treatment or inflammatory syndrome [17]. In our study; PC and PS deficiency was noted in $13.5 \%$ and $21.5 \%$ of cases respectively, results close to those of a Tunisian study and an Algerian series which found respectively $20.1 \%$ and $11 \%$ of patients with PC deficiency, $33.8 \%$ and $17.5 \%$ of cases with PS deficiency, unlike a study in France that targeted a prevalence of PC and PS deficiency in symptomatic patient groups in $3 \%$ and 3 to $6 \%$ of cases respectively, while its prevalence in the general population is between 0.003 and $0.13 \%$ for PS deficiency and only $0.3 \%$ for PC deficiency [17-18]. This discrepancy between our results and the literature data could probably be due to a high percentage of people with PC and/or PS deficiencies in the Maghreb population.

\subsubsection{The Search for Anti-phospholipid Antibodies}

As for the detection of APL antibodies, it was positive in $8 \%$ of patients, similar to an American study that showed the presence of lupus anticoagulants in $8 \%$ to $14 \%$ of patients with DVT [19]. The relative risk of thrombosis and thromboembolic recurrence is in the order of 10 and 3 respectively; this risk appears to be more correlated with the presence of lupus anticoagulants than with anticardiolipin [20]. Anticoagulant treatment may interfere with the detection of circulating anticoagulant, when the latter causes spontaneous prolongation of TCA and is confirmed by TCA with the addition of excess phospholipids (STACLOT LA).

\subsubsection{The Determination of Factor VIII}

For years, factor VIII testing was only used in familial hemophilia A. Recently; the Leiden team showed that the 
increase in factor VIII could be associated with an increased risk of venous thrombosis [21]. Other studies have shown that the increase in blood factor VIII levels in some patients who have had DVT persists over time and is not due to any known etiology [21-22-23-24]. This is a likely constitutional anomaly with a prevalence ranging from $1.4 \%$ to $4 \%$ in the general population, and from $6 \%$ to $14 \%$ in the population with a history of thrombosis [25]. In our study 7 patients had an elevation of factor VIII or $3.5 \%$ but the dosage of this factor was performed only in $7 \%$ of our sample which is not very representative.

\subsubsection{The Search for the Mutation of Factor V and Factor II}

this was the case for the mutation of F V and F II also which represented $3 \%$ and $1.5 \%$ of our patients respectively. According to the literature, their frequency in France in patients with a history of thrombosis is $20 \%$ for $\mathrm{F} \mathrm{V}$ mutation and $6.2 \%$ for F II mutation, while in Africa; these anomalies are rare [26-27-28]. In the case of resistance to activated protein $\mathrm{C}(\mathrm{PCa})$, which results in the absence of prolongation of activated cephalin time in the presence of $\mathrm{PCa}$, molecular biology research of FV Leiden remains essential to establish the heterozygous or homozygous character of the mutation; this distinction is very important because if the relative risk of thrombosis is three to eight in the case of heterozygosis for the mutation, it is 80 in the case of homozygosity and the risk of thrombotic recurrence is greater [29-30].

\subsubsection{The Therapeutic and Prognostic Impact of \\ Thrombophilia Research in Thromboembolic Disease}

Our study was limited to establishing the epidemiological profile of thrombophilia by reporting the various anomalies encountered in our study population, in addition; other studies were interested in evaluating the therapeutic and prognostic impact of thrombophilia research in thromboembolic disease. Indeed, the main problem in VTED patients is the assessment of the optimal duration of treatment and therefore of long-term anticoagulation. This is mainly based on the analysis of the risk of recurrence of thrombosis by counterbalancing the risk of haemorrhagic complications related to treatment. While inherited thrombophilias do not increase the risk of VTE recurrence with anticoagulant therapy, their role in stopping anticoagulation is controversial. However, survival is not impaired in thrombophilic patients. Pabinger et al. evaluated mortality in the multicentre prospective European Prospective Cohort on Thrombophilia (EPCOT) study and did not find any decrease in survival in patients with thrombophilia. These data suggest that hereditary thrombophilia should not alter the duration of anticoagulation since there is no change in long-term survival. In addition, there are other factors, mainly clinical factors, which strongly influence the risk of recurrence and must adjust the duration of anticoagulation. The most fundamental element to look for is a thrombosis triggering factor since the recurrence rates of VTED at 5 years are $40 \%$ and $16 \%$ respectively, whether the first episode is spontaneous or induced. Other clinical risk factors include advanced age at first episode, male sex, proximal nature of
DVT, occurrence of a pulmonary embolism, existence of at least 2 thromboembolic episodes, cancer, filter cellar or post-thrombotic syndrome. Paraclinical risk factors are also reported: persistence of residual thrombosis, high fibrinogen concentration, elevation of factor VIII, factor IX or D-dimers when anticoagulants are stopped. Thus, the genetic factor only intervenes as a second intention in the decision to anticoagulate in the long term and does not supplant clinical risk factors in VTED [31].

\section{Conclusion}

All current data tend to limit the thrombophilia outcome to very specific situations, such as in women with unexplained repeated miscarriages, and in young patients following an idiopathic thromboembolic event, particularly when the family history is suggestive of an inherited disorder and the location of thrombosis is unusual.

The thrombogenic risk factor most commonly found in our study was PS deficiency, in contrast to the predominance of $\mathrm{F}$ $\mathrm{V}$ mutation in Western countries, hence the importance of emphasizing that in clinical practice, the first-line thrombophilia assessment should always combine, in accordance with the latest recommendations: inhibitor deficiencies, F V and F II mutations, APL antibodies and possibly F VIII determination.

The thrombophilia assessment, in order to be interpretable and to reflect the epidemiological profile of these abnormalities in the population, must be carried out in accordance with the requirements of the pre-analytical phase.

Although its cost is high and its benefit in terms of therapeutic management is low; the desire of patients and doctors to highlight an etiology could explain the majority of thrombophilia tests performed; which under no circumstances should generate illegitimate concern among patients.

\section{References}

[1] Seligsohn U, Lubetsky A. Seligsohn U, Lubetsky A. Genetic susceptibility to venous thrombosis. N Engl J Med 2001; 344: 1222-31.

[2] Bauer KA. The thrombophilias: well-defind risk factors with uncertain therapeutic implications. Ann Intern Med 2001; 135: 367-73.

[3] Outuraud F. Durée optimale du traitement anticoagulant au décours d'une embolie pulmonaire. RevuedesMaladiesRespiratoires (2011) 28: 1265-1277.

[4] Connors JM. Thrombophilia testing and venous thrombosis. $N$ Engl J Med. 2017; 377 (12): 1177-87.

[5] Baglin T, Gray E, Greaves M, et al. Clinical guidelines for testing for heritable thrombophilia. Br J Haematol 2010; 149: 209-20.

[6] Nicolaides AN, Breddin HK, Carpenter P, et al. Thrombophilia and venous thromboembolism. Internationalconsensus statement. Guidelines according to scientific evidence. IntAngiol 2005; 24: 1-26. 
[7] Kearon C, Akl EA, Comerota AJ, et al. Antithrombotic therapyfor VTE disease: Antithrombotic Therapy and Prevention ofThrombosis, 9th ed: American College of Chest Physicians Evidence-Based Clinical Practice Guidelines. Chest 2012; 141: e419S-94S.

[8] Konstantinides SV, Torbicki A, Agnelli G, et al.2014 ESC guidelines on the diagnosis and management of acute pulmonary embolism. Eur Heart J 2014; 35: 3033-69.

[9] Pernod G, Biron-Andreani C, Morange PE, et al. Recommendations on testing for thrombophilia in venous thromboembolic disease: a French consensus guideline. $J$ Mal Vasc 2009; 34: 156-203.

[10] Emmerich J. How to assess thrombophilia and which one ?Rev Prat 2007; 57 (7): 714-5.

[11] Caprini JA, Goldshteyn S, Glase CJ, Hathaway K. Thrombophilia testing in patients with venous thrombosis. Eur $J$ Vasc Endovasc Surg 2005; 30: 550-5.

[12] Martinelli I, Battaglioli T, Razzari C, Manucci PM. Type and location of venous thromboembolism in patients with factor $\mathrm{V}$ Leiden or prothrombin G20210A and in those with no thrombophilia. J ThrombHaemost 2007; 5: 98-101.

[13] Righini M, Paris S, Le Gal G, Laroche JP, Perrier A. Bounameaux H. Clinical relevance of distal deep vein thrombosis. Review of literature data. ThrombHaemost 2006; 95: 56-64.

[14] J.-S. Allaina, P. Gueret b, T. Le Galloua, C. Cazalets a, A. Lescoat a, P. JegoaaService de médecine interne, CHU hôpital Sud, 16, boulevard de Bulgarie, 35203 Rennes, France.

[15] Pernod G B-AC, Morange PE, Boehlen F, Constans J, Couturaud F, Drouet L, Jude B, Lecompte T, Le Gal G, Trillot $\mathrm{N}$, Wahl DRecommandations pour la recherche de facteurs biologiques de risque dans le cadre de la maladie thromboembolique veineuse. Recommandations GEHT-SFMV 2008. J Mal Vasc 2009; 34: 90.

[16] Zmazen R, Guermazi S, KarouiMAssociation de deux facteurs de risque thrombotique: Facteur $\mathrm{V}$ leiden et hyperhomocystéinemie, à propos d'un cas. Pathologiebiologie 2006.

[17] Kottke-Marchant K, Duncan A. Antithrombindeficiency: issues in laboratory diagnosis. Arch Pathol Lab Med 2002; 126: 1326-36.

[18] Jude B., Susen, S., Trillot N., Zawadzki C. Les thrombophilies constitutionnelles, Laboratoire d'Hématologie, Hôpital Cardiologique, CHRU, Lille.

[19] Roux, A., Sanchez, O., \& Meyer, G. Quel bilan de thrombophilie chez un patient atteint de maladie veineuse thromboembolique? Réanimation, 17 (4), 355-362. doi: 10.1016/j.reaurg.2008.03.011.

[20] Pabinger I, Schneider B. Thrombotic risk in hereditary antithrombin III, protein $\mathrm{C}$, or protein $\mathrm{S}$ deficiency. A cooperative, retrospective study. Gesellschaft fur Thrombose-und Hamostaseforschung (GTH) study group on natural inhibitors. ArteriosclerThrombVascBiol 1996; 16: 742-8.

[21] Bontempo F. A. The Lupus Anticoagulant -An UpdateMay, 2001; Transfusion Medicine Updates.

[22] Galli M, et al. Lupus anticoagulants are stronger risk factors for thrombosis than anticardiolipin antibodies in the antiphospholipid syndrome: a systematic review of the literature. Blood 2003; 101 (5): 1827-32.

[23] Koster T, Blann AD, Briet E, Vandenbroucke JP, Rosendaal FR. Role of clotting factor VIII in effect of von Willebrand factor on occurrence of deep-vein thrombosis. Lancet 1995; 345: $152-5$.

[24] Kraaijenhagen RA, In't Anker PS, Koopman MMW, Reitsma $\mathrm{PH}$, Prins $\mathrm{MH}$, Van den Ende A, et al. High plasma concentration of factor VIIIc is a major risk factor for venous thromboembolism. ThrombHaemost 2000; 83: 5-9.

[25] O’Donnell J, Tuddenham EGD, Manning R, Kemball C, Johnson D, Laffan M. High prevalence of elevated factor VIII levels in patients referred for thrombophilia screening: role of increased synthesis and relationship to the acute phase reaction. ThrombHaemost 1999; 81: 680-3.

[26] O'Donnell J, Mumford AD, Manning RA, Laffan M. Elevation of FVIIIc in venous thromboembolism is persistent and independent of the acute phase response. ThrombHaemost 2000; 83: 10-3.

[27] Bloemenkamp KWM, Helmerhorst FM, Rosendaal FR, Vandenbroucke JP. Venous thrombosis, oral contraceptives and high factor VIII levels. Thromb Haemost 1999; 82: 10247.

[28] De Mitrio V, Marino R, Scaraggi FA, Di Bari L, Giannoccaro F, Petronelli $\mathrm{M}$, et al. Influence of factor VIII/von Willebrand complex on the activated protein $\mathrm{C}$ resistance phenotype and on the risk for venous thromboembolism in heterozygous carriers of the factor V Leiden mutation. Blood CoagulFibrinol 1999; 10: 409-16.

[29] Rees DC. World distribution of factor V Leiden, PMID: 7475606, [PubMed - indexed for MEDLINE], Lancet. 1995 Oct 28; 346 (8983): 1133-4.

[30] Rosendaal FR, Koster T, Vandenbroucke JP, Reitsma PH. High risk of thrombosis in patients homozygous forfactor $\mathrm{V}$ Leiden (activated protein $\mathrm{C}$ resistance).

[31] Allain J. S, Gueret. P, Le Gallou. T, Cazalets. C, Lescoat. A, Jego. PLa recherche de thrombophilie héréditaire et son impact thérapeutique dans la maladie thromboembolique veineuse: résultats d'une étude monocentrique rétrospective sur 162patientsLa Revue de Médecine Interne; Volume 36, Supplement 2, December 2015, Page A58. 